\title{
Imaging of pulmonary perfusion using subtraction CT angiography is feasible in clinical practice
}

\author{
Dagmar Grob $^{1}$ (D) Luuk J. Oostveen ${ }^{1} \cdot$ Mathias Prokop ${ }^{1} \cdot$ Cornelia M. Schaefer-Prokop $^{2} \cdot$ Ioannis Sechopoulos $^{1}$. \\ Monique Brink ${ }^{1}$
}

Received: 25 April 2018 / Revised: 24 July 2018 / Accepted: 28 August 2018 / Published online: 25 September 2018

(C) The Author(s) 2018

\begin{abstract} pretation, benefits and limitations of arterial subtraction CTPA.

\section{Key Points} distribution in the pulmonary parenchyma.

- SCT could have an added value in detection of pulmonary embolism.

\author{
Abbreviations \\ CT Computed tomography \\ CTPA Computed tomography pulmonary angiography \\ DECT Dual-energy computed tomography \\ DLP Dose length product \\ MRI Magnetic resonance imaging \\ PE Pulmonary embolism \\ SCT Subtraction computed tomography
}

Subtraction computed tomography (SCT) is a technique that uses software-based motion correction between an unenhanced and an enhanced CT scan for obtaining the iodine distribution in the pulmonary parenchyma. This technique has been implemented in clinical practice for the evaluation of lung perfusion in CT pulmonary angiography (CTPA) in patients with suspicion of acute and chronic pulmonary embolism, with acceptable radiation dose. This paper discusses the technical principles, clinical inter-

- SCT uses motion correction and image subtraction between an unenhanced and an enhanced CT scan to obtain iodine

- SCT requires only software implementation, making it potentially more widely available for patient care than dual-energy CT.

Keywords Subtraction technique $\cdot$ Computed tomography scanner $\cdot$ Contrast media $\cdot$ Perfusion imaging $\cdot$ Pulmonary embolism

\section{Introduction}

Imaging techniques have been developed over the years to study pulmonary perfusion, not only as a tool to investigate

Dagmar Grob

Dagmar.Grob@radboudumc.nl

1 Department of Radiology and Nuclear Medicine, Radboud University Medical Center, Geert Grooteplein 10, 6525 GA Nijmegen, The Netherlands

2 Department of Radiology and Nuclear Medicine, Meander Medical Centre, Maatweg 3, 3813 TZ Amersfoort, The Netherlands the sequelae of vascular obstruction, such as acute and chronic pulmonary embolism (PE), but lately also as a potential tool to characterise inflammation and the malignancy potential of lung lesions [1-3].

As early as 1964, nuclear medicine has been able to assess pulmonary perfusion using isotopes that accumulate in the capillary bed [4]. Although all modern nuclear medicine methods can accurately quantify true perfusion in the pulmonary parenchyma, they also have important drawbacks, such as a low spatial resolution, issues with isotope availability, production and handling, and high cost [5].

Magnetic resonance imaging (MRI) and computed tomography $(\mathrm{CT})$ are more widely available, but are not capable of imaging true substance exchange. Total intravascular movement can be measured with MRI using arterial spin labelling techniques. In addition, volume and speed of vascular contrast distribution are measured with dynamic magnetic resonance angiography [6]. MRI does not use ionising radiation, and has a better spatial resolution than nuclear imaging techniques, but its images are frequently technically inadequate because of a low signal-to-noise ratio, susceptibility and motion artefacts due to air and respiratory movement, and the low amount of tissue in the lungs [2,7]. Therefore, MRI is not used as a 
primary imaging tool of the pulmonary vasculature in most hospitals. CT is far more widely accepted as the modality of choice for evaluation of the lungs because of its higher spatial and temporal resolution [8,9] and its new ability to display iodine distribution, reflecting pulmonary perfusion.

A CT technique commonly used in clinical practice for assessing pulmonary perfusion is dual-energy CT (DECT). It uses material decomposition of iodine from other materials to visualise the regional pulmonary distribution of intravenous contrast in the pulmonary vessels, including the capillaries [10]. This is accomplished by almost simultaneously irradiating the patient with two x-ray beams of different energy, or by using spectral detectors and then processing the data to generate iodine maps, at a radiation dose similar to or moderately higher than CT pulmonary angiography (CTPA) [11]. This technique can show PE-associated perfusion defects (PD) $[12,13]$ in concordance with ventilation-perfusion single-photon emission computed tomography (V/Q SPECT) findings [14], with increased the sensitivity in detection of PE at CTPA $[12,15]$. However, DECT requires dedicated dual-energy hardware.

An alternative, yet far less widely implemented CT technique to evaluate pulmonary perfusion alongside CTPA is subtraction CT (SCT), made possible by a post-processing technique that does not require special hardware. This technical note will introduce the concept of SCT, along with its clinical interpretation, benefits, limitations, and future perspectives.

\section{Technical principle}

SCT involves the subtraction of an unenhanced, pre-injection CT image from an enhanced, post-injection CT image to obtain information on iodine distribution. Since every CT scanner is capable of making an unenhanced and enhanced CT scan, the technique would be more widely applicable than DECT. SCT was introduced by Screaton in 2003 and Wildberger in 2005 [16, 17]. Both studies showed clear visualisation of perfusion defects due to vascular obstruction in anesthetised animals, which guaranteed almost no motion between the two scans.

In phantom experiments with different iodine densities, SCT showed a higher contrast-to-noise ratio between soft tissue and iodine compared to DECT. In DECT, the signal difference between the low- and high-energy images is roughly linearly associated with the local iodine concentration. This implies that the signal of the high-energy image is actually subtracted from the low-energy image, reducing the signal in DECT compared to SCT, where the iodine signal is fully exploited [18, 19]. However, subject motion between the two acquisitions in SCT must be absent or compensated for to result in only the iodine signal. This means that motion correction is the biggest challenge in SCT. With increasing performance and speed of registration algorithms, achieving adequate motion correction is now feasible (Fig. 1). In our practice, we use motion correction software for SCT $\left({ }^{\text {SURE }}\right.$ Subtraction Lung, Canon Medical Systems) that employs an iterative, non-rigid registration framework to register the unenhanced to the enhanced CT images [20]. This means that both linear and non-linear voxel displacements can be registered. Experiments with dynamic digital phantoms demonstrated that this software can correct motion adequately. Specifically, in simulated scans involving a caudocranial diaphragm position difference of $20 \mathrm{~mm}$ between the unenhanced and enhanced scans, the $75^{\text {th }}$ percentile of the lung voxel-to-voxel residual error distance was $1.6 \mathrm{~mm}$ [21]. This seems sufficient for evaluating clinically relevant perfusion defects caused by segmental and potentially first-order subsegmental vascular obstructions, as perfusion defects caused by subsegmental embolism are in the centimetre range [16], and the median diaphragm difference between unenhanced and enhanced CT scans in our clinical practice is $5.7 \mathrm{~mm}$ in CTPA scans.

\section{Subtraction in clinical practice}

\section{Image acquisition}

SCT has been added to CTPA for all adult patients with suspicion of $\mathrm{PE}$ at our institution for the last 3 years. CT examinations are performed on a 320-multislice detector row CT system (Aquilion ONE GENESIS and VISION, Canon Medical Systems) according to the protocol in Table 1. In order to avoid DECT-like artefacts, it is crucial that the tube voltages for the enhanced and unenhanced scans are equal [22]. Both the unenhanced and the enhanced scan are acquired during a shallow breath-hold. To obtain an optimal CTPA enhancement, $60 \mathrm{ml}$ of intravenous contrast is administered with an iodine concentration of at least 300 $\mathrm{mg} / \mathrm{ml}$ and bolus triggering in the pulmonary artery, in order to guarantee sufficient contrast circulation in the pulmonary parenchyma.

\section{Radiation dose}

In our hospital, the median dose-length product (DLP) of this protocol in patients scanned between August 2016 and January 2017 ( $\mathrm{n}=354$ patients) was $191 \mathrm{mGy} \cdot \mathrm{cm}$ (mean DLP: $266 \mathrm{mGy} \cdot \mathrm{cm}$ ), with a median DLP of $59 \mathrm{mGy} \cdot \mathrm{cm}$ (mean: $74 \mathrm{mGy}^{\circ} \mathrm{cm}$ ) for the unenhanced scan and of 122 $\mathrm{mGy}^{\cdot} \mathrm{cm}$ (mean: $183 \mathrm{mGy} \cdot \mathrm{cm}$ ) for the enhanced scans. The effective dose was $2.8 \mathrm{mSv}$, which is calculated from the total median DLP multiplied by $0.0146 \mathrm{mSv} /(\mathrm{mGy} \cdot \mathrm{cm})$ [23]. This is lower than the average dose of a CTPA scan (3-5 mSv) [8]. In 55 patients who underwent both DECT and SCT in a prospective study, the whole subtraction protocol was executed 
Fig. 1 1-mm axial CTPA reconstructions after subtraction of an unenhanced CT from a CTPA with a diaphragm difference of $11 \mathrm{~mm}$ between the scans. a With motion correction, and (b) without motion correction

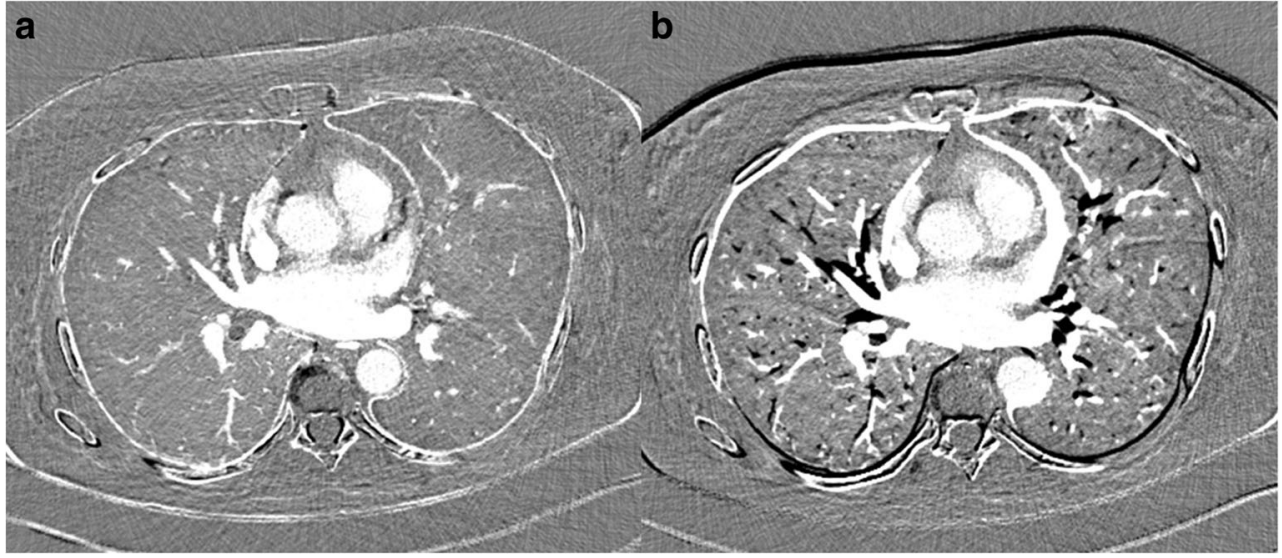

with a lower radiation dose than DECT, while subjectively evaluated image quality was better [24].

\section{Reconstruction}

The subtraction software automatically selects the unenhanced and enhanced scans, applies a mask to extract only the lung areas and registers and deforms the lungs in the unenhanced scan to their shape and position in the enhanced scan. After subtraction, it automatically generates 1$\mathrm{mm}$ greyscale iodine maps of the lungs with exclusion of the large vessels and 5-mm heat scale colour maps as an overlay on top of the CTPA images. These maps reflect true Hounsfield unit density differences in the pulmonary parenchyma between the two scans, with a pre-set WW/WL of 100/ 50 (Fig. 2). It is possible to reconstruct afterwards images at their own preference thickness, for example, even thicker slices, like $10 \mathrm{~mm}$.

\section{Interpretation}

Subtraction perfusion maps can only be used complementary to CTPA. Initial evaluation of the maps in three directions facilitates appreciation of the normal ventro-dorsal gradient of pulmonary blood volume in the supine patient [25], and easier recognition of artefacts. In addition, this initial review allows for a more accurate assessment of the shape of potential perfusion inhomogeneities [26], which allows for differentiation of the typical triangular shape of perfusion inhomogeneities due to vascular pathology or bronchopathy from pathologies such as emphysema (Fig. 3). To distinguish these entities, scrutiny of the CTPA is necessary to rule out PE, while evaluation of the bronchial system and lung parenchyma is necessary to rule out bronchial abnormalities or emphysema. Because perfusion inhomogeneities due to vascular or bronchial disease affect structures larger than the secondary lobules, which are within the centimetre range, reconstruction of thick multiplanar reconstructions of 5-10 $\mathrm{mm}$ or are sufficient
Table 1 Example of an SCTPA protocol

\begin{tabular}{|c|c|}
\hline Acquisition/injection & Specific settings \\
\hline Pre-contrast CT & $\begin{array}{l}\text { Exposure parameters: } 100 \mathrm{kV} \text {, automatic exposure control (SD 35) } \\
\text { Scan parameters: cranio-caudal scan with } 80 \times 0.5 \mathrm{~mm} \text { collimation, pitch } 0.8 \\
0.275 \text { s rotation time, shallow breath-hold } \\
\text { Reconstruction: } 1 \mathrm{~mm} \text { sections with } 0.8 \mathrm{~mm} \text { increment, } \\
\text { 3rd-generation iterative reconstruction (AIDR-3D enhanced) }\end{array}$ \\
\hline Contrast injection & $\begin{array}{l}60 \mathrm{ml} \text { iodinated contrast }(300 \mathrm{mg} / \mathrm{ml})+40 \mathrm{ml} \text { saline chaser @ } 5 \mathrm{ml} / \mathrm{s} \text { via a } 20 \mathrm{G} \text { needle } \\
\text { in the left arm }\end{array}$ \\
\hline Bolus triggering & $\begin{array}{l}\text { ROI placement on pulmonary trunk, level circa } 1 \mathrm{~cm} \text { below carina, absolute threshold: } \\
220 \mathrm{HU} \text {. After reaching the threshold there is } 5 \mathrm{~s} \text { scan delay. The related software for } \\
\text { automatic bolus triggering is }{ }^{\text {SURE }} \text { Start }\end{array}$ \\
\hline Post-contrast CT & $\begin{array}{l}\text { Exposure parameters: } 100 \mathrm{kV} \text {, automatic exposure control (SD 22.5) } \\
\text { Scan parameters: cranio-caudal scan with } 80 \times 0.5 \mathrm{~mm} \text { collimation, pitch } 0.8 \\
0.275 \text { s rotation time, shallow breath-hold } \\
\text { Reconstruction: } 1 \mathrm{~mm} \text { sections with } 0.8 \mathrm{~mm} \text { increment, } \\
\text { 3rd-generation iterative reconstruction (AIDR-3D enhanced) }\end{array}$ \\
\hline
\end{tabular}


Fig. 2 5-mm (a) axial and (b) sagittal reconstructions of a subtraction iodine map on top of CTPA of normally-perfused lungs in a supine position. Both reconstructions show a normal gravity-dependent gradient, in the ventro-dorsal and the craniocaudal direction

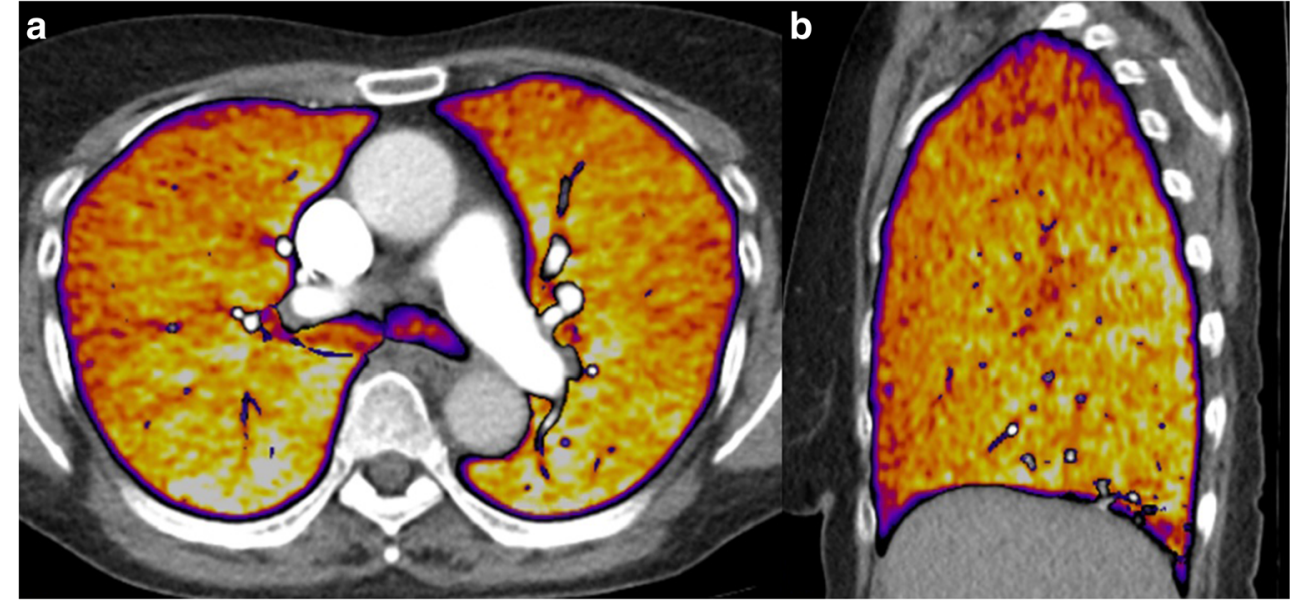

for initial interpretation. One-millimetre-thick slices can be used to assess smaller structures such as subsegmental PE, but at the expense of the increased noise and a higher susceptibility to the effects of inaccurate motion correction.

\section{Artefacts}

Beam-hardening artefacts may occur close to the high-density contrast column next to the superior caval vein in cases where a high-concentration of contrast material is still present in the injection veins [13]. These artefacts are less severe than those encountered in dual-source-based dual-energy techniques (Fig. 4) [24]. The probable reason is that the two tubes image the same structures with a time delay that is roughly a quarter of the rotation time, which might be enough for substantial variations in local contrast concentration in the inflow veins [13].

The images need to be evaluated in axial reconstruction but also in coronal or sagittal reconstructions to recognise artefacts. Motion artefacts can occur in SCT if lung volume and position, diaphragm position or cardiac pulsation significantly differ between the scans, even after registration. The type of artefact predominantly occurs in the basal lung fields, slightly above the diaphragm, and in the paracardiac region [21]. Presence of these artefacts can be assessed by checking differences between the enhanced and unenhanced scans on

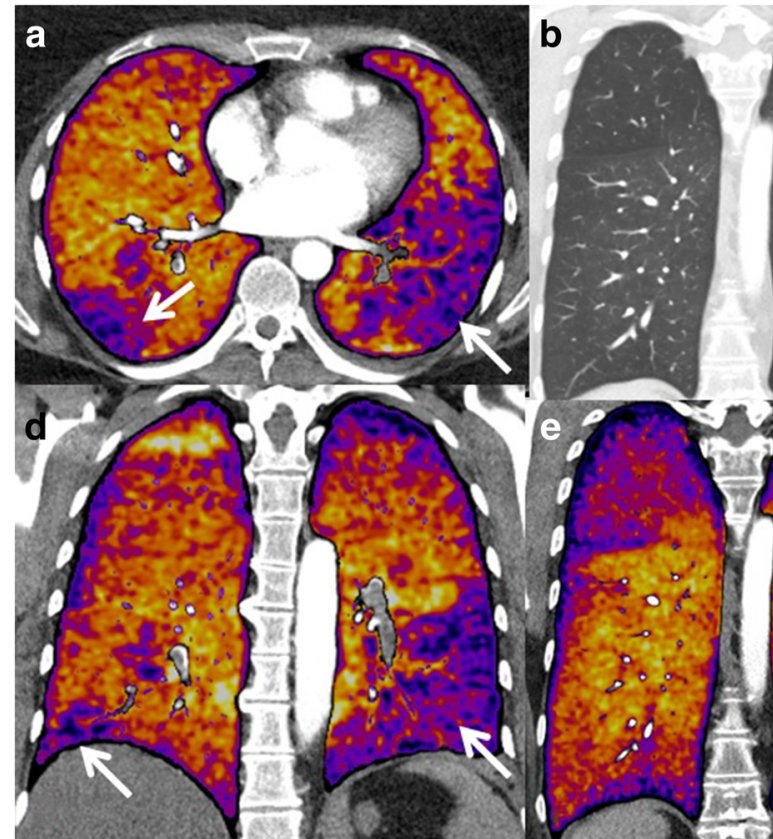

Fig. 3 a, d 3-mm slices of a patient with bilateral lobar pulmonary embolism and corresponding wedge-shaped perfusion defects in both lungs (arrows). b 3-mm slices of CTPA in a lung window, and, (e) a coronal view with iodine map of a patient with left lower lobe bronchopathy with mucous plugging (arrow) and

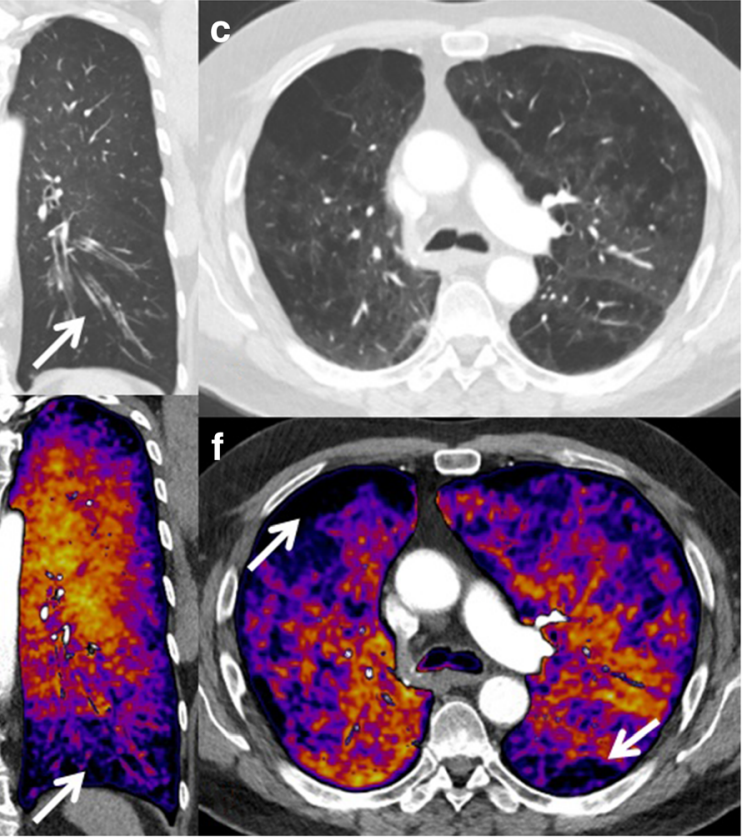

corresponding perfusion defects (arrows). c, f 3-mm reconstruction of CTPA and subtraction maps of a patient with predominant centrilobular emphysema; the destroyed pulmonary parenchyma does not show iodine uptake (arrows) 
Fig. 4 3-mm axial and coronal slices of subtraction iodine maps and dual-energy iodine maps, both obtained from a dual-source scanner. The arrows show typical beam-hardening artifacts that are more severe in dual-energy than in subtraction iodine maps

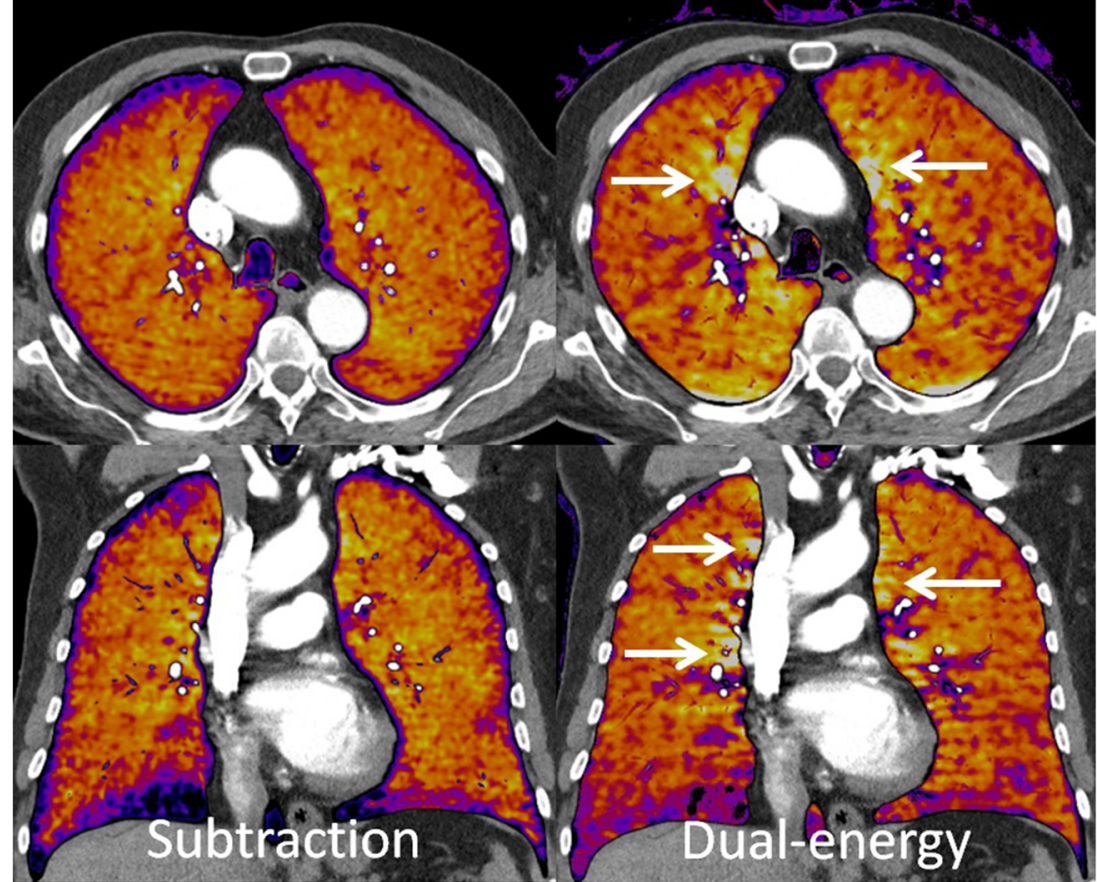

coronal reconstructions and producing 10-mm MinIP from the subtraction iodine maps. In case of severe motion correction flaws, black lines parallel to the vasculature will appear. The thicker the line, the worse the artefacts are.

Suboptimal contrast enhancement of the lung shows lowdensity areas in the iodine maps that can hamper the diagnostic evaluation. However, adapting the WW/WL of the greyscale and colour heat map can help.

\section{Discussion}

SCT is a simple, inexpensive and fast method for imaging and relative quantification of pulmonary perfusion at an acceptable radiation dose. The technique has been implemented in clinical practice in patients with suspicion of pulmonary embolism for several years, while having potential for other clinical applications that evaluate enhancement of the pulmonary parenchyma.

Iodine maps created with SCT are similar to those created with DECT. Artefact behavior is different, however; DECT images suffer from motion artefacts if some of the anatomy in the field of view moves during or between the high- or lowenergy scan. The latter effect is non-existent for energydiscriminating detectors, and negligible for a rapid $\mathrm{kV}$ switching system, but could play a role in dual-source systems or systems with dual rotation [18]. Motion artefacts in SCT occur if the patient moves during one of the two scans or if the inspirational difference between scans is major and is not adequately addressed. Streak artefacts due to contrast inflow are less severe with SCT compared to dual-source DECT [24].
SCT- and DECT-derived iodine maps reflect the iodine enhancement at the moment of image acquisition. However, absolute quantification of pulmonary perfusion parameters is impossible to achieve from a single acquisition, be it SCT or DECT, and requires sequential scanning (CT perfusion). This is because the variability in contrast enhancement among patients is high when a single acquisition is evaluated. The pulmonary contrast enhancement not only depends on the presence of pathology and contrast administration parameters, but also on parameters such as respiratory cycle dynamics, cardiac output, and bronchial physiology $[27,28]$. In addition, the enhancement pattern in the pulmonary parenchyma may change with time after contrast arrival, especially if there is bronchial arterial collateral supply to areas of the lung that are hypo-perfused by the pulmonary artery [29]. A second scan in the systemic arterial phase can help distinguish these two perfusion components. If sequential scanning were performed at a reasonable dose, absolute quantification might be possible. This might result in the ability to obtain functional (perfusion) information of structures in the pulmonary parenchyma. Other possibilities could be to characterise small structures or lesions, e.g. nodules, with the potential benefit of distinguishing between benign and malignant nodules [30].

Improvements in SCT could be achieved by reducing motion artefacts due to cardiac motion, pulsation and breathing in the pre- and post-contrast images using electrocardiogram (ECG) synchronisation or advanced motion-correction techniques. Improved registration of small vessels would provide not only subtraction imaging of the lung parenchyma, but of the vasculature itself [31]. This can be helpful for direct clot detection (lack of iodine enhancement causes a defect in the 
vessel on iodine maps). Improved beam hardening would help eliminate streak artefacts due to high contrast concentration in inflow veins [32].

In conclusion, software-based motion correction combined with temporal subtraction enables imaging of contrast enhancement in the lungs similar to DECT. The technique is widely applicable at a radiation dose equivalent to DECT. Similar to DECT, SCT depicts perfusion abnormalities in patients with vascular, bronchial or other pathology. Future advances in pulmonary SCT include dynamic acquisition and reduction of motion artefacts in small structures.

Funding Funding for this research has been provided by Canon Medical Systems, manufacturer of CT systems and developer of the algorithms evaluated in this study. The study data and results were generated and controlled at all times by the research personnel at Radboud University Medical Centre, with no influence from Canon.

\section{Compliance with ethical standards}

Guarantor The scientific guarantor of this publication is Monique Brink

Conflict of interest The authors of this manuscript declare relationships with the following company: Canon Medical Systems.

Statistics and biometry No complex statistical methods were necessary for this paper.

Informed consent Written informed consent was not required for this study because this technical note does not include a patient trial, but rather gives an overview of SCT.

Ethical approval Institutional review board approval was not required because this technical note does not include a patient trial, but rather gives an overview of SCT.

\section{Methodology}

- Performed at one institution

Open Access This article is distributed under the terms of the Creative Commons Attribution 4.0 International License (http:// creativecommons.org/licenses/by/4.0/), which permits unrestricted use, distribution, and reproduction in any medium, provided you give appropriate credit to the original author(s) and the source, provide a link to the Creative Commons license, and indicate if changes were made.

\section{References}

1. Chae EJ, Song JW, Krauss B et al (2010) Dual-energy computed tomography characterization of solitary pulmonary nodules. $\mathrm{J}$ Thorac Imaging 25:301-310

2. Hopkins SR, Wielpütz MO, Kauczor HU (2012) Imaging lung perfusion. J Appl Physiol (1985) 113:328-339

3. Mistry NN, Pollaro J, Song J, De Lin M, Johnson GA (2008) Pulmonary perfusion imaging in the rodent lung using Dynamic Contrast Enhanced MRI. Magn Reson Med 59:289-297
4. Elgazzar AH (2015) The Pathophysiologic Basis of Nuclear Medicine, 3 edn. Springer International Publishing, New York City

5. Dubsky S, Fouras A (2015) Imaging regional lung function: a critical tool for developing inhaled antimicrobial therapies. Adv Drug Deliv Rev 85:100-109

6. Haller S, Zaharchuk G, Thomas DL, Lovblad KO, Barkhof F, Golay X (2016) Arterial Spin Labeling Perfusion of the Brain: Emerging Clinical Applications. Radiology 281:337-356

7. Ley-Zaporozhan J, Ley S, Eberhardt R et al (2007) Assessment of the relationship between lung parenchymal destruction and impaired pulmonary perfusion on a lobar level in patients with emphysema. Eur J Radiol 63:76-83

8. Remy-Jardin M, Pistolesi M, Goodman LR et al (2007) Management of suspected acute pulmonary embolism in the era of CT angiography: a statement from the Fleischner Society. Radiology 245:315-329

9. Patel S, Kazerooni EA, Cascade PN (2003) Pulmonary embolism: optimization of small pulmonary artery visualization at multidetector row CT. Radiology 227:455-460

10. Johnson TR, Krauss B, Sedlmair M et al (2007) Material differentiation by dual energy CT: initial experience. Eur Radiol 17:1510 1517

11. Thieme SF, Johnson TR, Lee C et al (2009) Dual-energy CT for the assessment of contrast material distribution in the pulmonary parenchyma. AJR Am J Roentgenol 193:144-149

12. Pontana F, Faivre JB, Remy-Jardin M et al (2008) Lung perfusion with dual-energy multidetector-row CT (MDCT): feasibility for the evaluation of acute pulmonary embolism in 117 consecutive patients. Acad Radiol 15:1494-1504

13. Kang MJ, Park CM, Lee CH, Goo JM, Lee HJ (2010) Dual-energy CT: clinical applications in various pulmonary diseases. Radiographics 30:685-698

14. Meysman M, Everaert H, Buls N, Nieboer K, de Mey J (2015) Comparison of ventilation-perfusion single-photon emission computed tomography (V/Q SPECT) versus dual-energy CT perfusion and angiography (DECT) after 6 months of pulmonary embolism (PE) treatment. Eur J Radiol 84:1816-1819

15. Okada M, Kunihiro Y, Nakashima Y et al (2015) Added value of lung perfused blood volume images using dual-energy CT for assessment of acute pulmonary embolism. Eur J Radiol 84:172-177

16. Screaton NJ, Coxson HO, Kalloger SE et al (2003) Detection of lung perfusion abnormalities using computed tomography in a porcine model of pulmonary embolism. J Thorac Imaging 18:14-20

17. Wildberger JE, Klotz E, Ditt H, Spuntrup E, Mahnken AH, Günther RW (2005) Multislice computed tomography perfusion imaging for visualization of acute pulmonary embolism: animal experience. Eur Radiol 15:1378-1386

18. Faby S, Kuchenbecker S, Sawall S et al (2015) Performance of today's dual energy $\mathrm{CT}$ and future multi energy $\mathrm{CT}$ in virtual noncontrast imaging and in iodine quantification: A simulation study. Med Phys 42:4349-4366

19. Baerends E, Oostveen LJ, Smit CT et al (2018) Comparing dual energy $\mathrm{CT}$ and subtraction $\mathrm{CT}$ on a phantom: which one provides the best contrast in iodine maps for sub-centimetre details? Eur Radiol. https://doi.org/10.1007/s00330-018-5496-X

20. Goatman K, Plakas C, Schuijf J, Beveridge E, Prokop M (2014) Computed tomography lung iodine contrast mapping by image registration and subtraction. Proc of SPIE 9034, Medical Imaging 2014: Image Processing, https://doi.org/10.1117/12.2043551

21. Grob D, Oostveen LJ, Rühaak J et al (2018) under review Accuracy of registration algorithms in subtraction CT of the lungs: a digital phantom study

22. Winklhofer S, Lambert JW, Sun Y, Wang ZJ, Sun DS, Yeh BM (2016) Pelvic Beam-Hardening Artifacts in Dual-Energy CT Image Reconstructions: Occurrence and Impact on Image Quality. AJR Am J Roentgenol 208:114-123 
23. Deak PD, Smal Y, Kalender WA (2010) Multisection CT protocols: sex- and age-specific conversion factors used to determine effective dose from dose-length product. Radiology 257:158-166

24. Grob D, Smit EJ, Oostveen LJ et al (2017) Intra-individual comparison of direct subtraction vs. dual-energy for imaging of pulmonary perfusion- a feasibility study. European Congres of Radiology (ECR) 2017, Vienna

25. Almquist HM, Palmer J, Jonson B, Wollmer P (1997) Pulmonary perfusion and density gradients in healthy volunteers. J Nucl Med 38:962-966

26. Roach PJ, Bailey DL, Schembri GP, Thomas PA (2010) Transition from Planar to SPECT V/Q Scintigraphy: Rationale, Practicalities, and Challenges. Semin Nucl Med 40:397-407

27. Takx RAP, Henzler T, Schoepf UJ et al (2017) Predictive value of perfusion defects on dual energy CTA in the absence of thromboembolic clots. J Cardiovasc Comput Tomogr 11:183-187

28. Meinel FG, Graef A, Sommer WH, Thierfelder KM, Reiser MF, Johnson TR (2013) Influence of vascular enhancement, age and gender on pulmonary perfused blood volume quantified by dualenergy-CTPA. Eur J Radiol 82:1565-1570

29. Koike H, Sueyoshi E, Sakamoto I, Uetani M (2017) Clinical Significance of Late Phase of Lung Perfusion Blood Volume (Lung Perfusion Blood Volume) Quantified by Dual-Energy Computed Tomography in Patients With Pulmonary Thromboembolism. J Thorac Imaging 32:43-49

30. Ohno Y, Nishio M, Koyama H et al (2015) Solitary pulmonary nodules: Comparison of dynamic first-pass contrast-enhanced perfusion area-detector CT, dynamic first-pass contrast-enhanced MR imaging, and FDG PET/CT. Radiology 274:563-575

31. Murphy K, van Ginneken B, Reinhardt JM et al (2011) Evaluation of registration methods on thoracic CT: the EMPIRE10 challenge. IEEE Trans Med Imaging 30:1901-1920

32. Barrett JF, Keat N (2004) Artifacts in CT: recognition and avoidance. Radiographics 24:1679-1691 